\title{
On functions whose graph is of linear measure 0 on sets of measure 0
}

by

James Foran (Milwaukee, Wisc.)

Abstract. Denote the class of real valued functions whose graph is of linear measure 0 on sets of Lebesgue measure 0 by $\bar{N}$. Then Baire functions in $\bar{N}$ are shown to have $\sigma$-finite length graphs. If $G$ is generalized Lipschitz and $F$ belongs to $\bar{N}$, then $F+G, F \cdot G$, and $F \circ G$ belong to $\bar{N}$. Examples are given which show that the first result can not be improved to include all functions in $\bar{N}$ and that the second can not be improved to include absolutely continuous functions $G$.

Let $\bar{N}$ denote the class of real valued functions whose graph on any set of Lebesgue measure 0 is of linear measure 0 . In this paper the class $\bar{N}$ is compared to the class of functions which satisfy Lusin's condition $(N)$, i.e., functions for which the image of any set of measure 0 is of measure 0 .

On can readily observe that functions in $\bar{N}$ satisfy Lusin's condition (N). For let $F \in \bar{N}$, let $Z$ be a set of measure 0 , and let $B(F ; Z)$ denote the graph of $F$ on the set $Z$. Then given any $\varepsilon>0$ there exists a sequence of planar sets $\left\{A_{i}\right\}$ such that $B(F ; Z)=\bigcup A_{i}$ and $\sum \operatorname{diam} A_{i}<\varepsilon$. Hence $F(Z) \subset \operatorname{Proj}_{\mathrm{Y}} A_{i}$ and

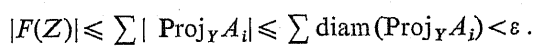

Thus $|F(Z)|=0$ and, since $Z$ is an arbitrary set of measure $0, F$ satisfies Lusin's condition $(\mathrm{N})$.

Since functions in $\bar{N}$ satisfy condition (N), continuous functions in $\bar{N}$ are differentiable on a set which has positive measure in every interval (cf. [4], p. 286). An important class of continuous functions which belong to $\bar{N}$ are the generalized absolutely continuous (ACG) functions, the primitives for the Denjoy integral. A continuous function $F$ is ACG if the line is a countable union of sets $E_{n}$ on each of which $F$ is absolutely continuous. To see that ACG functions belong to $\bar{N}$, let $E_{n}$ be a set of real numbers and suppose that $F$ is absolutely continuous on $E_{n}$; i.e., $\forall \varepsilon>0, \exists \delta>0$ such that $\sum\left|F\left(x_{i}\right)-F\left(x^{\prime}\right)\right|<\varepsilon$ whenever $\left\{\left[x_{i}, x_{i}^{\prime}\right]\right\}$ is any sequence of pairwise non-overlapping intervals with end points in $E_{n}$ and $\sum\left|x_{i}-x_{i}^{\prime}\right|<\delta$. Let $\varepsilon>0$ be given and $Z$ be any set of measure 0 . Cover $Z \cap E_{n}$ with a sequence of non-overlapping intervals $\left[x_{i}, x_{i}^{\prime}\right]$ with end points in $E_{n}$ such that $\sum\left|x_{i}-x_{i}^{\prime}\right|<\delta$, 
where $\delta<\varepsilon$ is determined by the definition of absolute continuity of $F$ on $E_{n}$. Then $B\left(F ; E_{n} \cap Z\right)$ can be covered by the sequence of sets $A_{i}=\left[x_{i}, x_{i}^{\prime}\right] \times\left[y_{i}, y_{i}^{\prime}\right]$ where $y_{i}$ and $y_{i}^{\prime}$ are respectively the infimum and supremum of $F(x)$ on the set $\left[x_{i}, x_{i}^{\prime}\right] \cap E_{n} \cap Z$. It follows that

$$
\sum \operatorname{diam} A_{i} \leqslant \sum\left|x_{i}-x_{i}^{\prime}\right|+\sum\left|y_{i}-y_{i}^{\prime}\right|<2 \varepsilon .
$$

Since $\varepsilon$ is arbitrary, the graph of $F$ on $E_{n} \cap Z$ is of linear measure 0 . Consequently, provided that $F$ is $A C G$, the graph of $F$ on $Z$ is of linear measure 0 and, since $Z$ is arbitrary, $F \in \vec{N}$.

Theorems 1 and 2 indicate properties which hold for functions in $\bar{N}$. The theorems are followed by examples that limit the possibilities of further generalization.

THEOREM 1. If $F$ is a Baire function and $F \in \bar{N}$ then the graph of $F$ is of $\sigma$-finite linear measure; i.e., the graph is the countable union of sets of finite linear measure.

Proof. Baire functions are characterized by the fact that their graphs are Borel sets (cf. [2], p. 300). Davies [1] showed that every analytic set of positive linear measure contains a closed subset of finite (non-zero) linear measure. Consequently, if $F$ is a Baire function and $B(F)$, the graph of $F$, is not of $\sigma$-finite measure, there is a closed set $E_{0} \subset B(F)$ such that $0<\Lambda\left(E_{0}\right)<\infty$. Suppose that for each $\gamma$ with $0 \leqslant \gamma<\alpha<\omega_{1}$ a closed set $E_{\gamma} \subset B(F)$ has been chosen such that $0<\Lambda\left(E_{\gamma}\right)<\infty$ and such that for every $\beta<\gamma, E_{\gamma} \cap E_{\beta}=\varnothing$. Then since $B(F)-\bigcup_{\gamma<\alpha} E_{\gamma}$ is a Borel set and $B(F)$ is not of $\sigma$-finite measure, it follows that $\Lambda\left(B(F)-\bigcup_{\gamma<\alpha} E_{\gamma}\right)=\infty$ and that there is a closed set $E_{\alpha} \subset B(F)-\bigcup_{\gamma<\alpha} E_{\gamma}$ such that $0<\Lambda\left(E_{\alpha}\right)<\infty$. In this manner one chooses an uncountable collection of sets $\left\{E_{\alpha}\right\}_{\alpha<\omega_{1}}$ which are closed, pairwise disjoint, contained in $B(F)$, and satisfy $0<A\left(E_{\alpha}\right)<\infty$. Let $E_{\alpha}^{\prime}=\operatorname{Proj}_{X}\left(E_{\alpha}\right)$. Since the line is not the union of uncountably many pairwise disjoint measurable sets of positive measure, (if it were, some interval $[k, k+1]$ would be the union of uncountably many measurable sets of measure $>1 / n)$ it follows that one of the $E_{\alpha}^{\prime}$ is of measure 0 .. But $\left|E_{\alpha}^{\prime}\right|=0$ and $\Lambda\left(E_{\alpha}\right)>0$ contradicts the fact that $F \in \bar{N}$ and thus the theorem is proved.

ExAmple 1. Assuming the continnum hypothesis, there exists a function $f \in \bar{N}$ whose graph is not of $\sigma$-finite linear measure.

Pro of. Let $G_{0}, G_{1}, \ldots, G_{\alpha}, \ldots, \alpha<\omega_{1}$ be a well-ordering of the $G_{\delta}$ measure 0 subsets of the reals. Let $H_{\gamma}=G_{\gamma}-\bigcup_{\gamma^{\prime}<\gamma} G_{\gamma^{\prime}}$. Let $F_{0}, F_{1}, \ldots, F_{\beta}, \ldots, \beta<\omega_{1}$ be a wellordering of the closed subsets of the plane whose projection on the $x$-axis is of positive measure. Let $E_{0}$ be the first closed subset to occur in the well-ordering, $\left\{F_{\beta} \mid G_{0} \cap \operatorname{Proj}_{X} F_{\beta} \neq \varnothing\right\}$. Select $\left(x_{0}, y_{0}\right) \in E_{0}$ such that $x_{0} \in G_{0} \cap \operatorname{Proj}_{X} E_{0}$. For each $x \in G_{0}$ define $f(x)=y_{0}$. Suppose that for every $\gamma<\alpha<\omega_{1}$, if $H_{\gamma} \neq \varnothing$, then $E_{\gamma}$ has been selected as the first closed subset of the well ordering

$$
\left\{F_{\beta} \mid F_{\beta} \neq E_{\gamma}, \forall \gamma^{\prime}<\gamma \text { and } H_{\gamma} \cap \operatorname{Proj} F_{\beta} \neq \varnothing\right\} \text {. }
$$

Suppose further that $\left(x_{\gamma}, y_{\gamma}\right) \in E_{\gamma}$ has been chosen with $x_{\gamma} \in H_{\gamma} \cap \operatorname{Proj}_{X} E_{\gamma}$ and that for every $x \in H_{\gamma}$, the function $f$ has been defined with $f(x)=y_{\gamma}$. Then for $\alpha<\omega_{1}$, if $H_{\alpha} \neq \varnothing$, it follows that the well-ordering,

$$
\left\{F_{\beta} \mid F_{\beta} \neq E_{\gamma} \forall \gamma<\alpha \text { and } H_{\alpha} \cap \operatorname{Proj} F_{\beta} \neq \varnothing\right\}
$$

is not empty. This is so because from the uncountable collection of $F_{\beta}$ whose projection intersects $H_{\alpha}$, an at most countable collection of $E_{\gamma}$ have been removed. Let $E_{\alpha}$ be the first member of the well-ordering and select $\left(x_{\alpha}, y_{\alpha}\right) \in E_{\alpha}$ so that $x_{\alpha} \in H_{\alpha}$. For $x \in H_{\alpha}$, define $f(x)=y_{\alpha}$. Thus the function $f(x)$ is defined inductively for all real numbers $x$.

To see that $f \in \bar{N}$, let $Z$ be a set of measure 0 on the line and let $G_{\alpha_{0}}$ be a $G_{\delta}$ subset which contains $Z$. Since $B(f ; Z) \subset B\left(f ; G_{\alpha_{0}}\right) \subset \bigcup_{\gamma \leqslant \alpha_{0}} H_{\gamma} \times\left\{y_{\gamma}\right\}$ and since $\left|H_{\gamma}\right|=0$ for each $\gamma$, it follows that $B(f ; Z)$ is of linear measure 0 .

To see that $B(f)$ is not of $\sigma$-finite linear measure let $F=F_{\beta}$ be a closed set of positive planar measure. Since $\operatorname{Proj}_{X} F$ contains uncountably many $G_{\delta}$ subsets of measure 0 and yet $\left\{F_{\gamma}\right\}_{\gamma<\beta}$ is at most countable, it follows that $F_{\beta}$ was selected as some $E_{\gamma_{0}}$ and consequently $\left(x_{\gamma_{0}}, y_{\gamma_{0}}\right) \in B(f) \cap F_{\beta}$. Since this is true for every closed set of positive planar measure, the complement of $B(f)$ does not contain any closed set of positive planar measure. Thus $B(f)$ is of full outer measure in the plane and thus is not of $\sigma$-finite linear measure because sets of $\sigma$-finite linear measure are of planar measure 0 .

THEOREM 2. If $G$ is a generalized Lipschitz function (i.e., if the line can be written as a countable union of sets on each of which $G$ is Lipschitz) and $F \in \bar{N}$, then $G+F \in \bar{N}$, $G \cdot F \in \bar{N}$, and $G \circ F \in \bar{N}$.

Proof. Let $G$ be a generalized Lipschitz function, let $F \in \bar{N}$, and let $Z$ be any set of measure 0 . Then the line can be written as the union of a sequence of sets $\left\{E_{k}\right\}$ such that $|G(x)|<k$ on $E_{k},|F(x)|<k$ on $E_{k}$, and $|G(x)-G(y)|<k|x-y|$ for each $x, y \in E_{k}$. Since $F \in \bar{N}$ it follows that $\Lambda\left(B\left(F ; Z \cap E_{k}\right)\right)=0$. Thus, given $k$ and $\varepsilon>0$, there is a sequence of sets $\left\{A_{n}\right\}$ such that $B\left(F ; Z \cap E_{k}\right)=\cup A_{n}$ and $\sum_{n} \operatorname{diam} A_{n}<\varepsilon$. Let $A_{n}^{\prime}=\operatorname{Proj}_{X} A_{n}$. Then

$$
\sum_{n} \operatorname{diam} A_{n}^{\prime}<\varepsilon \quad \text { and } \quad|G(x)-G(y)|<k|x-y| \quad \text { for each } x, y \in A_{n}^{\prime} \text {. }
$$

Concerning the sum, $G+F$, it follows that

$$
B\left(G+F ; Z \cap E_{k}\right)=\bigcup_{n} B\left(G+F ; A_{n}^{\prime}\right)
$$

and

$$
\begin{aligned}
& \sum_{n} \operatorname{diam} B\left(G+F ; A_{n}^{\prime}\right) \\
& \quad \leqslant \sum_{n}\left(\sup _{n} F(x)+\sup _{n} G(x)-\inf _{n} F(x)-\inf _{n} G(x)\right)+\sum_{n} \operatorname{diam} A_{n}^{\prime},
\end{aligned}
$$

3 - Fundamenta Mathematicae XCVI 
where $\sup _{n}$ and $\inf _{n}$ are the supremum and infimum taken on the sets $A_{n}^{\prime}$. Thus

$\sum_{n} \operatorname{diam} B\left(G+F ; A_{n}^{\prime}\right)$

$$
\begin{aligned}
& \leqslant \sum_{n}\left(\sup _{n} F(x)-\inf _{n} F(x)\right)+\sum_{n}\left(\sup _{n} G(x)-\inf _{n} G(x)\right)+\sum_{n} \operatorname{diam} A_{n}^{\prime} \\
& \leqslant \sum_{n} \operatorname{diam} A_{n}+k \sum_{n} \operatorname{diam} A_{n}^{\prime}+\sum_{n} \operatorname{diam} A_{n}^{\prime} \\
& \leqslant 2 \varepsilon+k \varepsilon .
\end{aligned}
$$

Concerning the product, $G \cdot F$, it is sufficient to consider the set $E_{k}^{\prime}=E_{k} \cap$ $\cap(G \geqslant 0) \cap(F \geqslant 0)$. Similar arguments hold for the cases where $G \geqslant 0$ and $F<0$, $G<0$ and $F \geqslant 0, G<0$ and $F<0$. Then

$$
B\left(G \cdot F ; Z \cap E_{k}^{\prime}\right)=\bigcup_{n} B\left(G \cdot F ; A_{n}^{\prime} \cap E_{k}^{\prime}\right)
$$

and

$$
\sum_{n} \operatorname{diam} B\left(G \cdot F ; Z \cap E_{k}^{\prime}\right)
$$

$$
\leqslant \sum_{n}\left(\sup _{n} F(x) \cdot \sup _{n} G(x)-\inf _{n} F(x) \cdot \inf _{n} G(x)\right)+\sum_{n} \operatorname{diam} A_{n}^{\prime}
$$

where $\sup _{n}$ and $\inf _{n}$ are the supremum and infimum taken on the sets $A_{n}^{\prime} \cap E_{k}^{\prime}$. Thus

$$
\begin{aligned}
& \sum_{n} \operatorname{diam} B\left(G \cdot F ; A_{n}^{\prime} \cap E_{k}^{\prime}\right) \\
& \leqslant \sum_{n} \sup _{n} F(x)\left(\sup _{n} G(x)-\inf _{n} G(x)\right)+\sum_{n} \inf _{n} G(x)\left(\sup _{n} F(x)-\inf _{n} F(x)\right)+ \\
& \quad+\sum_{n} \operatorname{diam} A_{n}^{\prime} \\
& \leqslant k^{2} \cdot \sum_{n} \operatorname{diam} A_{n}^{\prime}+k \cdot \sum_{n} \operatorname{diam} A_{n}+\sum_{n} \operatorname{diam} A_{n}^{\prime} \\
& \leqslant k^{2} \varepsilon+k \varepsilon+\varepsilon .
\end{aligned}
$$

Concerning the composition, $G \circ F$, it follows that

$$
B\left(G \circ F ; Z \cap E_{k}\right)=\bigcup_{n} B\left(G \circ F ; A_{n}^{\prime}\right)
$$

and

$$
\begin{aligned}
\sum_{n} \operatorname{diam} B\left(G \circ F ; A_{n}^{\prime}\right) & \leqslant \sum_{n}\left(\operatorname{diam} G\left(F\left(A_{n}^{\prime}\right)\right)+\operatorname{diam} A_{n}^{\prime}\right) \\
& \leqslant \sum_{n}\left(k \cdot \operatorname{diam} F\left(A_{n}^{\prime}\right)+\operatorname{diam} A_{n}^{\prime}\right) \\
& \leqslant k \varepsilon+\varepsilon .
\end{aligned}
$$

Since $\varepsilon>0$ is arbitrary, the linear measure of the graphs of $G+F, G \cdot F$, and $G \circ F$ on $Z \cap E_{k}$ is 0 and, this being true for each $k$, the linear measure of the graphs of these functions on $Z$ is also 0 . Since $Z$ was an arbitrary set of measure 0 , it follows that $G+F, G \cdot F$, and $G \circ F$ all belong to $\bar{N}$ and the theorem is proved.

In neither of the three cases of Theorem 2 can generalized Lipschitz be improved to include absolutely continuous functions. This is true even if the $F \in \bar{N}$ are restricted to continuous functions. Mazurkiewicz [3] constructed a continuous function $f(x)$ on $[0,1]$ such that for $b \neq 0$ the function $f(x)+b x$ does not satisfy Lusin's condition (N). Thus it follows from Theorem 2 that Mazurkiewicz function does not belong to $\bar{N}$. However, this function does satisfy Banach's condition $\mathrm{S}$ and hence is the composition of two absolutely continuous functions (cf. [4], p. 288). Since absolutely continuous functions belong to $\bar{N}$ it follows that Theorem 2 can not be improved to include the composition of absolutely continuous functions with continuous functions belonging to $\bar{N}$.

The lemma that follows is preliminary to the construction of an increasing absolutely continuous function $\psi$ and a continuous function $\varphi \in \bar{N}$ such that their sum does not satisfy condition (N). Then Theorem 2 implies that $F(x)=e^{\varphi(x)} \in \bar{N}$. But $G(x)=e^{\psi(x)}$ is then an increasing function which satisfies condition $(\mathrm{N})$ and hence $G(x)$ is absolutely continuous. Clearly, $F \cdot G(x)=e^{\varphi(x)+\varphi(x)}$ does not satisfy condition $(\mathrm{N})$. It follows that Theorem 2 can not be improved to include the sum or product of absolutely continuous functions with continuous functions belonging to $\bar{N}$.

LEMMA 1. Let $F(x)$ be a continuous function which satisfies Lusin's condition $(\mathrm{N})$ on an interval I. Let $P \subset I$ be a perfect set of measure 0 . Then there exists a strictly increasing function $G(x)$ defined on $I$ such that $G$ and $G^{-1}$ are absolutely continuous and $\Lambda\left(B\left(F \circ G^{-1} ; G(P)\right)\right)=0$.

Proof. Since $F(P)$ is a compact set of measure 0 , there is a perfect set $P^{\prime}$ such that $P^{\prime} \times F(P)$ is of linear measure 0 . For example, $P^{\prime}$ can be constructed as follows: Let $Q=F(P), \varepsilon_{1}=\operatorname{diam} Q$, and $n_{1}=1$. Since $Q$ is a compact set of measure 0 , $\varepsilon_{i+1}$ and $n_{i+1}$ can be defined inductively so that $Q$ can be covered by $n_{i+1}$ intervals of equal length $\varepsilon_{i+1}$ such that $n_{i+1} \cdot \varepsilon_{i+1}<\varepsilon_{i} / 2^{i+1}$. Let $P^{\prime}$ be the set of all numbers $x$ of the form $\sum_{i} a_{i} \cdot \varepsilon_{i} ; a_{i}=0$ or 1 . Then $P^{\prime}$ can be covered by $2^{i+1}$ intervals of length $\varepsilon_{i+1}$ and hence $P^{\prime} \times Q$ can be covered by $n_{i+1} \cdot 2^{i+1}$ squares of diameter $\varepsilon_{i+1} \sqrt{2}$. Since $n_{i+1} \cdot 2^{i+1} \cdot \varepsilon_{i+1} \cdot \sqrt{2}<\varepsilon_{i} \cdot \sqrt{2}$ and since $\varepsilon_{i}$ tends to 0 , it follows that $P^{\prime} \times Q$ is of linear measure 0 .

Since $P^{\prime}$ is a perfect set, there is a strictly increasing function $G(x)$ such that $G(P)=P^{\prime}$ and such that $G(x)$ is linear on intervals contiguous to $P$. Then, since $G$ and $G^{-1}$ are strictly increasing and satisfy condition (N), both $G$ and $G^{-1}$ are absolutely continuous. Since $B\left(F \circ G^{-1} ; G(P)\right)$ is contained in $P^{\prime} \times F(P)$ it follows that $\Lambda(B(F \circ G ; G(P)))=0$ and the lemma is proved.

Mazurkiewicz function $f(x)$ is linear on the intervals contiguous to a perfect set $P$ of measure 0 and $f(x)+x$ fails to satisfy condition $(\mathrm{N})$ on $P$. Lemma 1 applied to $f(x)$ and $P$ yields a function $g(x)$. Let $\psi=g^{-1}$ and $\varphi=f \circ g^{-1}$. Then $\psi$ is increasing and absolutely continuous. Since $\Lambda\left(B\left(\varphi ; P^{\prime}\right)\right)=0$ and $\varphi$ is linear on the 
intervals contiguous to $P^{\prime}$, it follows that $\varphi \in \bar{N}$. If $u \in P^{\prime}$ and $g^{-1}(u)=x \in P$, then $(\psi+\varphi)(u)=g^{-1}(u)+f\left(g^{-1}(u)\right)=x+f(x)$. But then the image of $\psi+\varphi$ on the set $P^{\prime}$ is the same as the image of $f(x)+x$ on the set $P$. Since $P^{\prime}$ is of measure 0 and $f(x)+x$ fails to satisfy condition $(\mathrm{N})$ on $P$, it follows that $(\psi+\varphi)\left(P^{\prime}\right)$ is a set of positive measure, $\psi+\varphi$ does not satisfy condition $(\mathrm{N})$, and thus $\psi+\dot{+} \varphi \notin \bar{N}$.

\section{References}

[1] R. O. Davies, Subsets of finite measure in analytic sets, Indag. Math. 14 (1952), pp. 488-489.

[2] K. Kuratowski, Topology, New York-London-Warszawa 1966

[3] S. Mazurkiewicz, Sur les fonctions qui satisfont à la condition (N), Fund. Math. 16 (1930), pp. $348-352$.

[4] S. Saks, Theory of the Integral, second revised edition, New York 1937.

UNIVERSITY OF WISCONSIN-MILWAUKEE

Milwaukee, Wisconsi

\section{Demi-groupes, espaces affines et categories gauches}

par

André Batbedat (Montpellier)

Résumé. On étudie la structure déterminée sur un ensemble par une loi de composition binaire: $(a, b) \rightarrow a b$, qui vérifie: $(a b) c=b(a c)$ pour tous $a, b, c$.

Propriétés duales pour: $(a b) c=a(c b)$

Application aux notions d'espace affine gauche et de catégorie gauche.

Introduction. On sait qu'une loi de composition binaire est associative si elle vérifie pour tous $a, b, c$ :

(A): $(a b) c=a(b c)$.

Dans [7], V. G. Lemlein a considéré le cas:

$(\mathrm{AM}):(a b) c=c(b a)$

Nous nous plaçons ici dans l'hypothèse d'associativité gauche:

$(\mathrm{AG}):(a b) c=b(a c)$.

Les résultats se transposent dualement pour l'associativité droite: $(\mathrm{AD}):(a b) c=a(c b)$.

Mathématiquement une telle étude présente un grand intérêt car (A), (AG) et $(A D)$ sont les seuls cas où les applications qui à un élément associent ses translations intérieures au sens de [4] sont chacune un morphisme ou un antimorphisme.

En pratique nous verrons que bien souvent l'associativité gauche implique l'associativité.

Dans ce contexte nous précisons les concepts d'espace affine gauche et de catégorie gauche.

I. Généralités. Nous présentons succinctement dans ce chapitre quelques définitions et propriétés dont nous aurons besoin par la suite (pour plus de détails, consulter [1], [2], [3], [4] ou [8]).

I.1. Un binaire ([1] ou [2]) est un ensemble muni d'une loi de composition binaire: $(a, b) \rightarrow a b$. On note sans parenthèses le composé dans l'ordre écrit de gauche à droite (c'est ainsi que: $(a b) c$ est simplement noté: $a b c$ ).

Un demi-groupe est un binaire associatif (introduction) 